\title{
Clinico-Demographic, Hematological, and Comorbidity Profile of COVID-19 Patients Admitted at Tertiary Care Center
}

\author{
Hemant Kumar, Sumeet Dixit', Shobhit Shakya², Manoj Kumar Pandey, Nikhil Gupta², Preeti Gupta ${ }^{3}$, Shyam Murari Kalra, Amiya Pandey \\ Departments of Respiratory Medicine, ${ }^{1}$ Community Medicine and ${ }^{2}$ General Medicine, Dr. Ram Manohar Lohia Institute of Medical Sciences, ${ }^{3}$ Department of \\ Ophthalmology, Hind Institute of Medical Sciences, Lucknow, Uttar Pradesh, India
}

\section{Abstract}

Introduction: Severe acute respiratory syndrome coronavirus 2 has caused a worldwide pandemic. This study was aimed to describe the clinico-demographic, hematological, and comorbidity profile in a group of coronavirus disease 2019 (COVID-19) patients at a tertiary care center in north India. Materials and Methods: We conducted a prospective, single-center collection of data regarding clinico-epidemiological, hematological parameters, and comorbidity profile of COVID-19 patients admitted at a tertiary care facility. Results: Data from 200 patients with COVID-19 were collected and analyzed. The median age of the patients was 48 years, with $63 \%$ males, and $78 \%$ patients were from urban area. Ten patients were smokers while 11 patients were alcoholic. The occupation of $20.6 \%$ of patients was related directly or indirectly with medical or allied professions. The most common mode of transmission of COVID-19 was direct close contact with microbiologically confirmed patients. Fever $(n=93 ; 46.5 \%)$ was the most common presenting symptom and the median duration of onset of symptoms before admission were 3.5 days (range $2-6$ days). In hematological profile, lymphopenia $(n=48 ; 24 \%)$ had been the most common documented finding. Comorbidities were present in $39(19.5 \%)$ patients, of which diabetes mellitus $(n=25 ; 12.5 \%)$ was the most common. Furthermore, the case fatality rate in our study was $1.5 \%$. Conclusion: Among all patients of COVID-19 at our center, the characteristic findings included high proportion of male patients with younger to middle-age group, diabetes as most common comorbidity and people who were directly or indirectly in contact with the health-care system were more prone for developing the disease.

Keywords: Clinico-demographical, comorbidity, coronavirus disease 2019, reverse transcription polymerase chain reaction

\section{INTRODUCTION}

At the end of December 2019, a novel coronavirus was identified as the cause of a cluster of pneumonia cases in Wuhan, China, that has spread worldwide as a global pandemic. In February 2020, the World Health Organization designated the disease as coronavirus disease 2019 (COVID-19). The virus that causes COVID-19 is termed as severe acute respiratory syndrome coronavirus 2 . As per the WHO data, as on October 26, 2020, a total of 43,341,451 confirmed cases and $1,157,509$ deaths had been reported worldwide. As of October 25, 2020, the Ministry of Health and Family Welfare, India, has confirmed 7,864,811 COVID-19 cases and 118,534 deaths constituting second highest COVID-19 affected country after the United States. The clinico-demographic profile and outcomes of patients with COVID-19 have been variable in different countries. ${ }^{[1]}$ It is important to analyze and document the clinical presentation and comorbidity associated with

\begin{tabular}{|l|l|}
\hline \multicolumn{3}{|c|}{ Access this article online } \\
\hline Quick Response Code: & Website: \\
\hline & www.ijrc.in \\
\hline
\end{tabular}

this novel disease in the Indian population. The objective of this paper is to analyze the clinical spectrum of the patients ranging from their age, sex, travel history, clinical symptoms, laboratory evaluation, and associated comorbidity.

\section{Materials and Methods}

All microbiologically confirmed Covid-19 patients admitted at Dr. Ram Manohar Lohia Institute of Medical Sciences (Dr. RMLIMS), Lucknow, from March 1, to submission

Address for correspondence: Dr. Manoj Kumar Pandey, Assistant Professor, Department of Tuberculosis and Chest, Government Medical College, Azamgarh, Uttar Pradesh, India. E-mail: manojpandeyjnp2@gmail.com

This is an open access journal, and articles are distributed under the terms of the Creative Commons Attribution-NonCommercial-ShareAlike 4.0 License, which allows others to remix, tweak, and build upon the work non-commercially, as long as appropriate credit is given and the new creations are licensed under the identical terms.

For reprints contact:WKHLRPMedknow_reprints@wolterskluwer.com

How to cite this article: Kumar H, Dixit S, Shakya S, Pandey MK, Gupta N, Gupta P, et al. Clinico-demographic, hematological, and comorbidity profile of COVID-19 patients admitted at tertiary care center. Indian J Respir Care 2021;10:213-5.

Received: 03-01-2021 Revised: 08-02-2021

Accepted: 06-03-2021 Published: 14-06-2021 
of paper, were enrolled for the study. Written informed consent was obtained from all patients. Ethical approval was obtained from the ethical committee of the institution. Dr. RMLIMS is one of the dedicated COVID-19 care facility in Lucknow. Our center is designated as a referral facility (Level 3) for all COVID-19 patients from nodal public and private sector hospital, as per the government policy. All suspected patients of COVID-19 received at screening area, undergone reverse transcription polymerase chain reaction for COVID-19 pneumonia. All microbiologically confirmed COVID-19 cases were triaged into isolation facility, ward, high dependency unit or intensive care unit as per their clinical assessment. Data on epidemiological, demographic, clinical feature, travel history, addiction, laboratory, and associated comorbidity were obtained with data collection forms electronic medical records and history given by patients. Data on symptom onset and resolution were recorded. Time elapsed between the onset and resolution of symptoms was taken as time to clinical resolution. Further laboratory evaluation and treatment protocol was followed as per the Government of India Ministry of Health and Family Welfare guideline on COVID-19 pneumonia.

\section{RESULTS}

A total of 200 patients were studied, of whom $126(63 \%)$ were male and $74(37 \%)$ patients were female. The age-wise distribution of COVID-19 patients is shown in Table 1. Among 200 patients, 156 (78\%) patients were from urban area while $44(22 \%)$ were from rural area with $10 \%$ patients have some kind of addiction in form of alcohol consumption, tobacco chewing or smoking. The occupation of $20.6 \%$ of patients was related directly or indirectly with medical or allied professions. Close contact with COVID-19 patients $(n=140 ; 70 \%)$ constituted the most common mode of transmission. Other modes of transmission include foreign travel to an affected country $(n=5 ; 2.5 \%)$, and household close contact with a known COVID-19 patient $(n=70 ; 35 \%)$. Among all admitted patients, the most common symptoms were fever $(n=93 ; 46.5 \%)$; dry cough $(n=73 ; 36.5 \%)$; dyspnea $(n=44 ; 22 \%)$; body ache (n $54 ; 27 \%)$; tiredness $(n=34 ; 17 \%)$; throat irritation $(n=20 ; 10 \%)$; and nasal symptoms $(n=16 ; 8 \%)$. The median duration of onset of symptoms before admission was 3.5 days (ranges from 2 to 6 days).

Baseline hematological and biochemical parameters are presented in Table 2. Anemia was present in $85(42.5 \%)$ patients with mean hemoglobin of $12.59 \mathrm{~g} / \mathrm{dl} .15(7.5 \%)$ patients had leukopenia (total leucocyte count $[$ TLC] $<4000 / \mu 1$ ) while leukocytosis (TLC $>11,000 / \mu \mathrm{l}]$ was present in $19(9.5 \%)$ patients with mean polymorphs of $58.1 \%$ (ranges between $10 \%$ and $93 \%$ ) and mean lymphocyte of $29.8 \%$ (ranges between $3 \%$ and $76 \%$ ). Thrombocytopenia was present in $69(34.5 \%)$ patients with mean platelet count of $2.04 \mathrm{lakh} / \mathrm{cumm}$. Alanine aminotransferase levels were increased in $140(70 \%)$ with more than five times in 5 patients. Aspartate aminotransferase levels were increased in $87(43.5 \%)$ with more than five times in 2 patients. Comorbidities were present in $39(19.5 \%)$ patients, of which diabetes mellitus $(n=25 ; 12.5 \%)$ was the most common comorbidity observed, while $12(6 \%)$ patients were suffering from both diabetes and hypertension. Comorbidity profile of COVID-19 patients is shown in Table 3. The treatment was given as per the Ministry of Health and Family Welfare guideline on COVID-19 pneumonia. Among

\begin{tabular}{lc}
\hline $\begin{array}{l}\text { Table 1: Age-wise distribution of coronavirus disease } \\
\text { 2019 patients }\end{array}$ \\
\hline Age (years) & $\boldsymbol{n}(\%)$ \\
\hline$<10$ & $2(1)$ \\
$10-20$ & $17(8.5)$ \\
$20-30$ & $73(36.5)$ \\
$30-40$ & $39(19.5)$ \\
$40-50$ & $33(16.5)$ \\
$50-60$ & $25(12.5)$ \\
$>60$ & $11(5.5)$ \\
\hline
\end{tabular}

\begin{tabular}{|c|c|c|}
\hline & Mean \pm SD & Range \\
\hline $\mathrm{Hb}(\mathrm{g} \%)$ & $12.59 \pm 2.37$ & $3.96-17.1$ \\
\hline WBC (cells/cumm) & $7.16 \pm 3.96$ & $3.02-25.1$ \\
\hline RBC (cells/cumm) & $4.27 \pm 0.83$ & $1.62-7.8$ \\
\hline Platelets (lakhs/cumm) & $2.04 \pm 1.18$ & $0.56-8.44$ \\
\hline $\operatorname{MCV}(\mathrm{fl})$ & $90.52 \pm 12.88$ & $29-123$ \\
\hline $\mathrm{MCH}$ (pg/cell) & $30.03 \pm 4.41$ & $18-43$ \\
\hline $\mathrm{MCHC}(\mathrm{g} / \mathrm{dl})$ & $33.01 \pm 4.79$ & $23-76$ \\
\hline Polymorphs (\%) & $58.1 \pm 15.99$ & $10-93$ \\
\hline Lymphocytes (\%) & $29.80 \pm 13.48$ & $3-76$ \\
\hline Eosinophils (\%) & $2.438 \pm 0.37$ & $0-13$ \\
\hline Monocytes (\%) & $8.24 \pm 4.63$ & $0-40$ \\
\hline Serum_urea $(\mathrm{mg} \%)$ & $22.97 \pm 9.16$ & $4.8-47$ \\
\hline Creatinine (mg \%) & $0.83 \pm 1.19$ & $0.32-12.27$ \\
\hline Bilirub_total (mg \%) & $0.80 \pm 1.46$ & $0.15-14.8$ \\
\hline Bilirub_direct (mg \%) & $0.24 \pm 0.68$ & $0-6.8$ \\
\hline $\begin{array}{l}\text { Alkaline Phosphatase } \\
\text { (IU/L) }\end{array}$ & $90.01 \pm 56.59$ & $36-387$ \\
\hline
\end{tabular}

Table 3: Comorbidity profile of coronavirus disease 2019 patients

\begin{tabular}{lc}
\hline Comorbidity & $\boldsymbol{n}(\mathbf{\%})$ \\
\hline Diabetes & $25(12.5)$ \\
Hypertension & $21(10.5)$ \\
Chronic kidney disease & $2(1)$ \\
Coronary artery disease & $9(4.5)$ \\
Tuberculosis & $2(1)$ \\
Chronic obstructive pulmonary disease/asthma & $1(0.5)$ \\
Human immunodeficiency virus infection & $2(1)$ \\
Immunocompromised & $2(1)$ \\
\hline
\end{tabular}


132 symptomatic patients, $65(49.2 \%)$ became asymptomatic in $<5$ days, 39 (29.5\%) between 5 and 10 days and $28(21.2 \%)$ became asymptomatic more than 10 days after commencement of treatment. Three out of 200 admitted patients died during course of treatment accounting case fatality rate of $1.5 \%$. Diabetes was found to be the most common comorbidity among all deaths.

\section{Discussion}

We reported the epidemiological profile, clinical characteristics, laboratory, and comorbidity profile of 200 COVID-19 patients admitted to dedicated COVID care hospital of Dr. RMLIMS, Lucknow. In our study, the median age of COVID-19 patients was 48 years which was closest to study done by Huang et $a l . .^{[2]}$ (49.0 years) and Guan et al. ${ }^{[1]}$ (47 years). Majority of COVID-19 infected patients were male (63\%) which was accordant to the study done by Huang et al. and Chen et al. (73.0\%). This male predominance may have happened due to the fact that the majority of our patients were from hotspot area, created by public gathering, and mainly attended by males. Among 200 admitted COVID-19 patients, $10(5 \%)$ patients were smokers, and $11(5.5 \%)$ were alcoholic. According to a recent systematic review and meta-analysis, smoking appears to be a risk factor for COVID-19 progression with higher prevalence of smoking among COVID-19 patients with severe, progressive disease, or intensive care admission. ${ }^{[3]}$ The most common mode of transmission of COVID-19 virus in our patients was direct (in $70 \%$ patients) which was accordant to most studies done across the globe. Among all admitted patients, fever was the most common symptom ( $n=93 ; 46.5 \%)$ followed by dry cough $(n=73 ; 36.5 \%)$ which was similar to that reported by Huang et al. (91.7\%) and Wang et al. (87.9\%). In our study, lymphopenia had been the most common ( $n=48 ; 24 \%$ ) documented findings on complete blood count panel for the patients with COVID-19 infection among all hematological variables and this finding in our study correlates with various previous studies conducted elsewhere. In our study, the most common comorbidity was diabetes mellitus $(n=25 ; 12.5 \%)$ while hypertension was second-most common $(n=21 ; 10.5 \%)$. The less common comorbidities were coexisting infection with HIV $(n=2 ; 1 \%)$, renal disorders $(n=2 ; 1 \%)$, immunodeficiencies $(n=2 ; 1 \%)$, active tuberculosis $(n=2$; $1 \%)$, and COPD $(n=1 ; 0.5 \%)$. In contrary to our study, most of the previous meta-analysis had demonstrated hypertension as the most common associated comorbidity in the COVID-19 patients. ${ }^{[4]}$ Furthermore, the case fatality in our study was $1.5 \%$ (3/200) and the trend was closest to study done by Guan et al..$^{[1]}$ from China and Mohan et al..$^{[5]}$ from India.

\section{Conclusion}

COVID-19 disease is a very infectious disease with most common route of transmission through direct contact. The disease is more prevalent in young adult and middle age group, history of addiction and with history of diabetes. People who were directly or indirectly in contact with the health-care system are more prone to contract the disease.

\section{Financial support and sponsorship}

Nil.

\section{Conflicts of interest}

There are no conflicts of interest.

\section{RefEREnCes}

1. Guan WJ, Ni ZY, Hu Y, Liang WH, Ou CQ, He JX, et al. Clinical characteristics of coronavirus disease 2019 in China. N Engl J Med 2020;382:1708-20.

2. Huang C, Wang Y, Li X, Ren L, Zhao J, Hu Y, et al. Clinical features of patients infected with 2019 novel coronavirus in Wuhan, China. Lancet 2020;395:497-506.

3. Vardavas CI, Nikitara K. COVID-19 and smoking: A systematic review of the evidence. Tob Induc Dis 2020;18:20.

4. Gold MS, Sehayek D, Gabrielli S, Zhang X, McCusker C, Ben-Shoshan M. COVID-19 and comorbidities: A systematic review and meta-analysis. Postgrad Med 2020;132:749-55.

5. Mohan A, Tiwari P, Bhatnagar S, Patel A, Maurya A, Dar L, et al. Clinico-demographic profile \& hospital outcomes of COVID-19 patients admitted at a tertiary care centre in north India. Indian J Med Res 2020;152:61-9. 